Scientific Journal of October 6 University

ISSN (Print): 2314-8640

ISSN (Electronic): 2356-8119

Published by October 6 University (C) All Rights Reserved

Available online at: http:// sjou.journals.ekb.eg

Original Article
Citation: Tolba et al., (2016). Study of CRS 5 DOF Jacobian and Simulation of it's Singularity Using GA.. Sci.J. of Oct. 6 Univ. 3(1), 5965.

Copyright: (C) 2016 Tolba et al., This is an open-access article distributed under the terms of the Creative Commons Attribution License, which permits unrestricted use, distribution, and reproduction in any medium, provided the original author and source are credited.

\title{
Study of CRS 5 DOF Jacobian and Simulation of it's Singularity Using GA
}

\author{
Farid A. Tolba ${ }^{1}$, Magdy M. Abdelhameed ${ }^{1}$ and Mohammed Marey ${ }^{2}$ and Michael Nasr Shenouda ${ }^{1}$ \\ ${ }^{1}$ Mechatronics Engineering Department Ain Shams University Cairo, Egypt \\ ${ }^{2}$ Faculty of computer \& information sciences, Ain Shams University, Cairo, Egypt
}

Received: 8-11-2014/ Revised: 14-12-2014 / Accepted: 05-11-2015

\begin{abstract}
In this paper we will be discussing the singular configuration of CRS arm robot. Usually, in an open control scheme it is not that important to study your arm robot singularity as you can adjust your path as needed but in our visual servoing application which is considered a closed control scheme, it is very important to study your arm singularity and thus be able to avoid it or control the arm around these points.
\end{abstract}

Key Words: Singular configuration, CRS arm robot, servoing and singularity.

\section{Introduction}

The International Federation for the Promotion of Mechanism and Machine Science has defined a mechanism to be; 'a system of bodies designed to convert motions of, and forces on, one or several bodies into constrained motions of, and forces on, other bodies $^{\text {(1) }}$. An arm robot can be considered as a mechanism consists of a series of segments, jointed or sliding relative to one another, for the purpose of grasping and/or moving objects (pieces or tools) usually in several degrees of freedom. The components of a manipulator are connected by joints of various kinds: re-volute $(\mathrm{R})$, slider or prismatic $(\mathrm{P})$, screw or helical $(\mathrm{H})$, ball or spherical (S), planar (E). Singularity is defined as the configuration where the values of joint position cause the Jacobian to become singular or when the manipulator loses degree of freedom. The degenerate direction is defined as the degree of freedom that the manipulator loses in the singularity or a motion that the manipulator cannot execute while in singularity. Loss of freedom and thus loss of control are the main issues why we study the arm robot singular point and try to avoid them as possible. Many efforts were made to find the singularity of a specific arm robot, and to derive the conditions identify the properties of the singularity. some researchers used the screw theory or coordinate transformations ${ }^{(2-5)}$,other used a geometrical approach which we will depend on it mainly ${ }^{(6-8)}$ Gosselin and Angeles identified the different classes of mechanisms with respect of their singularities ${ }^{(6)}$ Zlatanov and al further redefined their concepts ${ }^{(9,10)}$ Hayes and Husty studied the singular configurations of wrist-partitioned $6 \mathrm{R}$ serial robots in general, and the KUKA KR-15/2 industrial robot in particular ${ }^{(11)}$.

\section{Related work}

Many have used Genetic Algorithms (GA) in determining the best path for a robot in terms of least time and torque to achieve the requested task without any collision with the robot or any external obstacles. GA are population based stochastic and global search methods that help in reaching a global solutions which are difficult to be found by normal methods ${ }^{(12)}$ Tabandeh and Clark presented a Genetic Algorithm for solving the inverse kinematics of a serial robotic manipulator which is capable of finding multiple solutions of the inverse kinematics $^{(13)}$. Kazem and Mahdi and Qudah optimized the point-to-point trajectory planning for a 3-link (redundant) robot arm using GA is to minimizing traveling time and space, while not exceeding a maximum predefined torque, without collision with any obstacle in the robot work space $^{(14)}$.

\section{Work}

In the following sections; the CRS arm robot structure will be shown in section (1), robot arm jacbian matrix calculation in section (2), then discussing robot arm singularity in general showing CRS singular points section (3).

\section{A. Robot arm structure}

The CRS is a 5DOF robot arm, Commands are issued to the robot system from program 
applications or terminal commands, or through the teach pendant. The complete system comes with a C500C controller and the teach pendant. The arm robot consists of 5 rotating joints with 5 separate axis of rotation; The $\mathrm{DH}$ parameters representing the robot arm is;

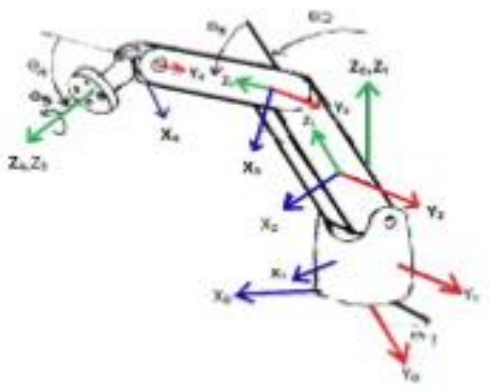

Fig. 1. CRS 5 DOF robot arm structure

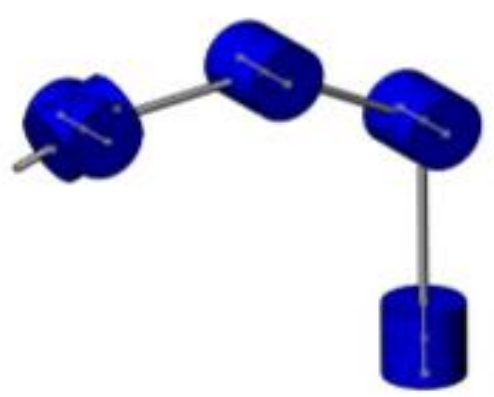

Fig. 2. CRS 5 DOF robot arm structure

The robot arm can be controlled through an open architecture mode where the C500C controller which sends currents directly to the motors. The user is responsible for implementing a stable feedback system with the feedback from the encoders.

\section{B. Robot jacobian matrix}

The velocity relationships, relating the linear and angular velocities of the end effector to the joint velocities are determined by the Jacobian of the forward kinematic equations.There is a need for kinematic analysis to study singularity of a robot manipulator.Kinematic analysis represents the jacobian matrix which is the best tool to analyze singularity of the robot. The equation which represents such relations is;

$$
\mathrm{v}=\mathrm{J} \cdot{ }^{*} \mathrm{Q}
$$

Where $\mathrm{v}$ represents the velocities of the end effector both linear and rotational velocities, while $\mathrm{Q}^{*}$ represents the change in every joints angle with respect to time, so we can write $\mathrm{v}$ and $\mathrm{Q}^{*}$ as follow

Table I CRS DH Parameters

\begin{tabular}{|c|c|c|c|c|}
\hline Link & Alpha & Angle offset & Theta & Distance \\
\hline 1 & 0 & 0 & 0 & 0 \\
\hline 2 & $\pi / 2$ & 0 & $\Theta 1$ & 254 \\
\hline 3 & 0 & 254 & $\Theta 2$ & 0 \\
\hline 4 & 0 & 254 & $\Theta 3$ & 0 \\
\hline 5 & $-\pi / 2$ & 0 & $\Theta 4$ & 0 \\
\hline 6 & 0 & 0 & $\Theta 5$ & 50.8 \\
\hline
\end{tabular}

$$
\begin{gathered}
v=\left[\begin{array}{c}
\dot{x} \\
\dot{y} \\
\dot{z} \\
\omega_{x} \\
\omega_{y} \\
\omega z
\end{array}\right] \\
\dot{Q}=\left[\begin{array}{c}
\dot{\theta_{1}} \\
\cdot \\
\dot{\theta_{n}}
\end{array}\right]
\end{gathered}
$$

Where $\mathrm{n}$ represents the number of joints. The Jacobian matrix is a size of $\mathrm{m} * \mathrm{n}$, where $\mathrm{m}$ identifies the number of degrees of freedom for the arm robot while $n$ represents the number of joints within the arm robot, from that we can conclude that Each row in the Jacobian matrix represents the effect of every joint on every coordinate of the end-effector. There are two ways to calculate Jacobian matrix, first one is the analytical jacobian and the second one is the geometrical Jacobian.First, the analytical Jacobian is computed via differentiation of the direct kinematics function with respect to the joint variables and it is used whenever it is necessary to refer to differential quantities of variables defined in the operational space. Consider Homogeneous transformation matrix $H(\theta)$ as in equation (4).

$$
H(\theta)_{n}^{0}=\left[\begin{array}{cc}
R(\theta)_{n}^{0} & T(\theta)_{n}^{0} \\
0 & 1
\end{array}\right]
$$

Where $R(\theta)^{0}{ }_{n}$ is the rotation matrix from the end effector to the base frame, and $T(\theta)_{n}^{0}$ is the transitional vector from the end effector to the base frame. As the robot moves about, both the joint variables $\theta_{\mathrm{n}}$ and the end-effector position $\mathrm{T}(\theta)_{\mathrm{n}}^{0}$ and orientation $R(\theta)^{0}{ }_{n}$ will be functions of time.

$$
v=\frac{\delta H(\theta)}{\delta \theta} \cdot\left[\begin{array}{c}
\dot{\theta}_{1} \\
\cdot \\
\dot{\theta_{n}}
\end{array}\right]
$$

The second way is the geometrical Jacobian and is calculated as follow, consider;

$$
J_{n}^{0}=\left[\begin{array}{l}
J_{v}^{0} \\
J_{\omega}^{0}
\end{array}\right]
$$


Where $\mathbf{J}^{0}{ }_{v}$ represents the time variant relation between linear velocities and $\theta$, and $\mathbf{J}^{0}{ }_{\omega}$ represents same one between angular velocities and $\theta$. so in case of revolute joints $\mathrm{J}_{v}$ can be calculated as;

$$
J_{v_{i}}=z_{i-1} \times\left(O_{n}-O_{i-1}\right)
$$

where $\mathrm{z}_{\mathrm{i}-1}$ is the vector representing the axis of rotation of each joint, and $\mathrm{On}$ or $\mathrm{O}_{\mathrm{i}-1}$ represents the origin point for each frame at each joint and $\mathbf{J}_{\omega}$ can be obtained through;

$$
J_{\omega}=z_{i-1}
$$

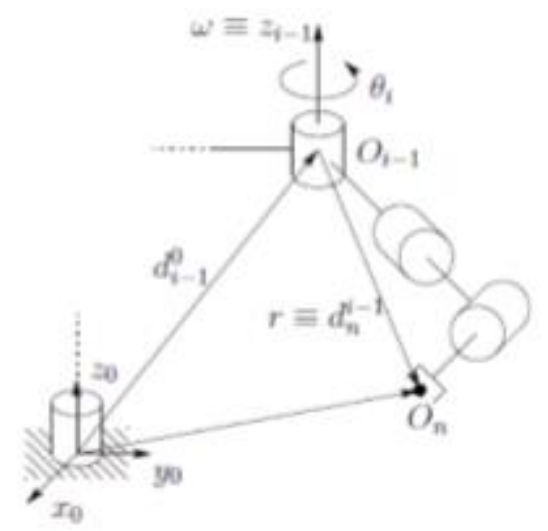

Fig. 3. Calculating Geometrical Jacobian

C. Robot Singularity analysis Singularity is defined as the configuration where the values of joint position cause the Jacobian to become singular or when the manipulator loses degree of freedom, that's mean a loss of instantaneous motion for the end-effector of one or more degrees, in addition to loss of control near singular points, where jacobian matrix is ill-conditioned and either the control algorithm fails or the joint velocities and accelerations may become unsustainably great and those points are reasons behind the arise of importance of singularity analysis. There are three well known singular points for an arm robot; first, the wrist singularity is when the first and third axes of the robots wrist (i.e. robot's axes 4 and 6) to line up then second wrist axis then attempts to spin 360 in zero time to maintain the orientation of the end effector. second, shoulder singularity and occurs when the wrist center lies on a cylinder that is centered about axis 1 and with radius equal to the distance between axes 1 and 4 .

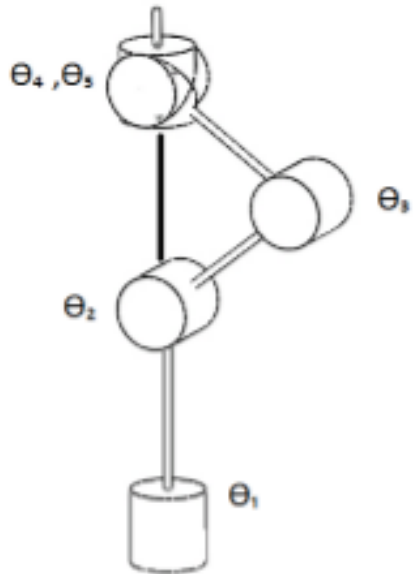

Fig. 4. Shoulder Singularity

Third, and it is known as elbow singularity and it occurs when the wrist's center lies in the same plane as axes 2 and 3, in other words when links 2 and 3 are one the same line.

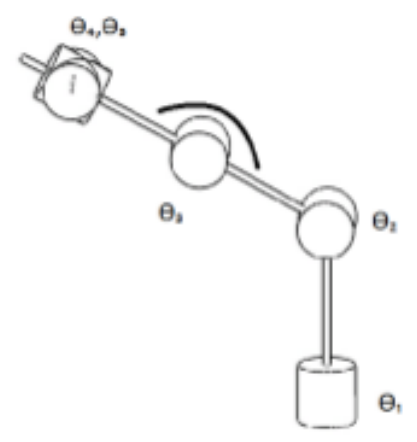

Fig. 5. Elbow singularity

D. CRS robot singularity analysis in this section the analytical condition of the CRS 5 DOF robot will be determined to use it in the numerical analysis phase to discover the different singularities attached to CRS robot.

1) Analytical study for CRS singularity condition: first; the joints vector to be defined;

$$
Q=\left[\begin{array}{l}
\theta_{1} \\
\theta_{2} \\
\theta_{3} \\
\theta_{4} \\
\theta_{5}
\end{array}\right]
$$

Jacobian usually calculated and obtained around the base frame of the robot, but the crs robot exerts the translation movement around the base frame while the orientation of the end effector is exerted around the end effector frame and the robot control uses same method so which will be used in the jacobian calculations as mentioned and as in equation(6) the CRS jacobian can be obtained as follow; 


$$
J_{C R S}=\left[\begin{array}{lllll}
J_{1,1} & J_{1,2} & J_{1,3} & J_{1,4} & J_{1,5} \\
J_{2,1} & J_{2,2} & J_{2,3} & J_{2,4} & J_{2,5} \\
J_{3,1} & J_{3,2} & J_{3,3} & J_{3,4} & J_{3,5} \\
J_{4,1} & J_{4,2} & J_{4,3} & J_{4,4} & J_{4,5} \\
J_{5,1} & J_{5,2} & J_{5,3} & J_{5,4} & J_{5,5}
\end{array}\right]
$$

the main concern is where the jacobian loses rank, in other words the configuration when determent of the jacobian tends to zero;

$$
\left|J_{\text {crs }}\right|=0
$$

results were not enough to study the whole work space of the robot and the various configuration of it. that's why numerical analysis was needed to fulfill such gap. 2) Numerical analysis of CRS robot: We studied the whole workspace of the robot arm to find any other singular point rather than the three known singular configuration, but we did not find any other point in the whole workspace, we studied the workspace with steps of 1 degree and 12 degree but there where no singular points except near the elbow singularity, we tried to study the shoulder singularity but due to some mechanical limitation in the arm robot such configuration can not be reached, and our robot is only 5 joints so wrist singularity is not applicable in our case also

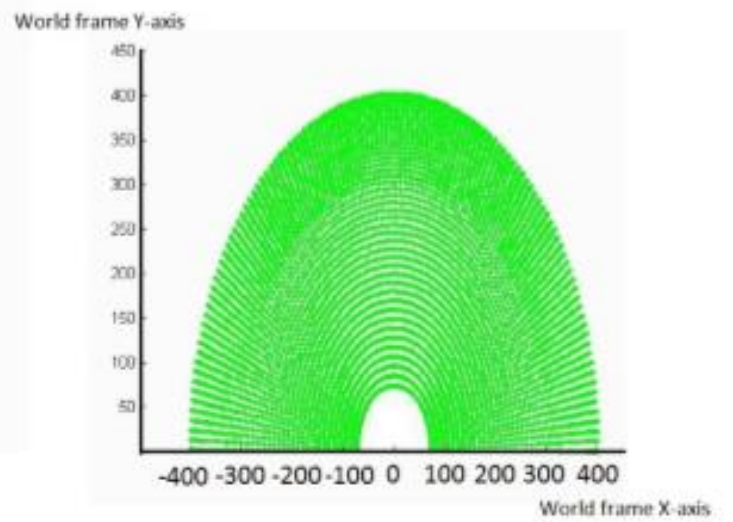

Fig. 6. $\mathrm{X}-\mathrm{Y}$ workspace plane

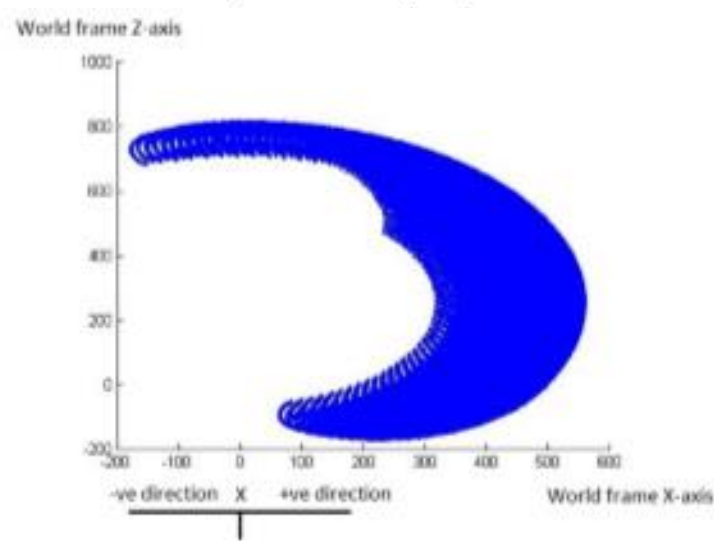

Fig. 7. X-Z workspace plane

later, we tried to focus on the area around the elbow singular points, so we studied the values of the determent from $\theta_{3}=0^{\circ}$ (where elbow singularity occurs) to $\theta_{3}=0.5^{\circ}$ with step of but no disturbance or malfunctioning of any type occurred in such range of degrees, so we tried to narrow the range we are looking in, so we check the singularity between $\theta_{3}=0^{\circ}$ and $\theta_{3}=0.1^{0}$ with steps 0.0001 we found a relation between the value of determent and the value of $\theta 3$ with only one step that's mean at $\theta 3=$ $0.0001 \circ$ the determent value jumped to 34.5 and by the fourth step the value of the determent was 125 which means it is far from a singular point or singular range. A graph for such values can be found below;

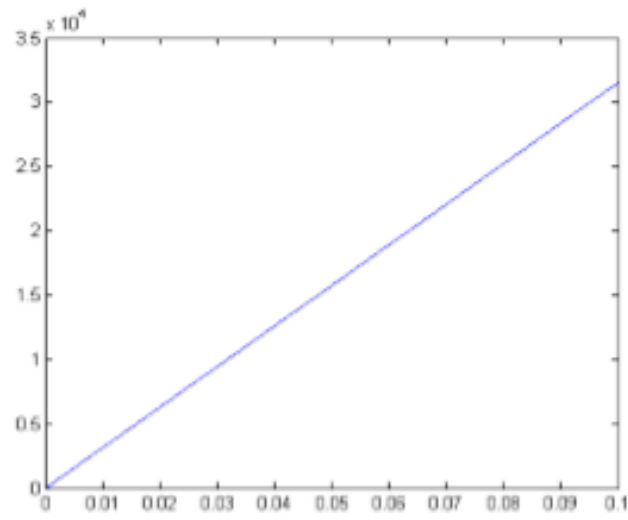

Fig. 8. the variation of determent values with respect to $\theta_{3}$ values

we tried to force the arm robot to reach elbow singular point to check it's response, and the response was an aggressive motion of the joints in the opposite direction that can me fatal in operation while holding a tool. The graph below shows such motion It is very clear in the second graph representing the

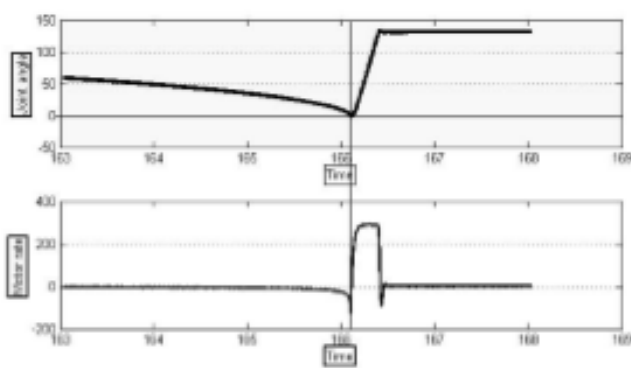

Fig. 9. The Motion of $\theta_{3}$ passing into singularity and motor rates

velocity of the motor controlling $\theta 3$ has been subjected to fatal overshoot in the reverse direction of the current motion. The importance of studying singularity arises also from the point of the effect of joint passing by or in a singular is that it affects the neighbor joints as well depending on the structure of the robot and mechanical control. The disturbance in motion included also $\theta_{2}$ and $\theta_{4}$, both joints where subject to reverse rates and forces that oppose the current motion. 

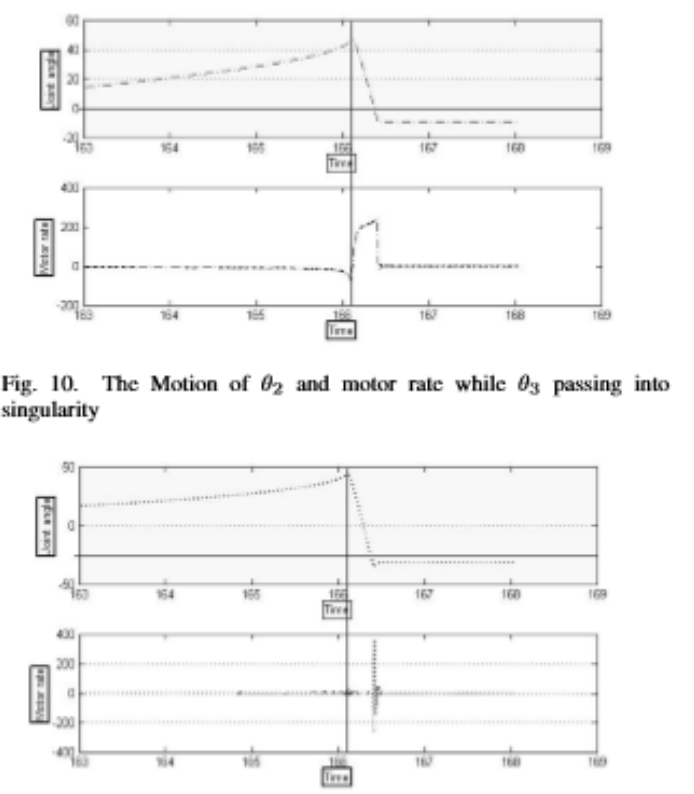

Fig. 11. The Motion of $\theta_{4}$ and motor rate while $\theta_{3}$ passing into singularity

E. Optimizing joints using GA Genetic algorithms (GAs) are global, parallel, stochastic search methods, founded on Darwinian evolutionary principles. it has arisen from a desire to model the biological processes of natural selection and population genetics $^{(15)}$. GA woks on any given objection function to find the best chromosome through promoting the best and elite ones in a series of successive generations by a given criteria of selection using a fitness function, each chromosome contains a defined number of genes, number of genes is the total number of variables for the fitness function and each chromosome is a possible solution for it. The worst chromosomes are neglected in the next generations and new chromosomeare substituted through defined operators named as mutation and cross-over.

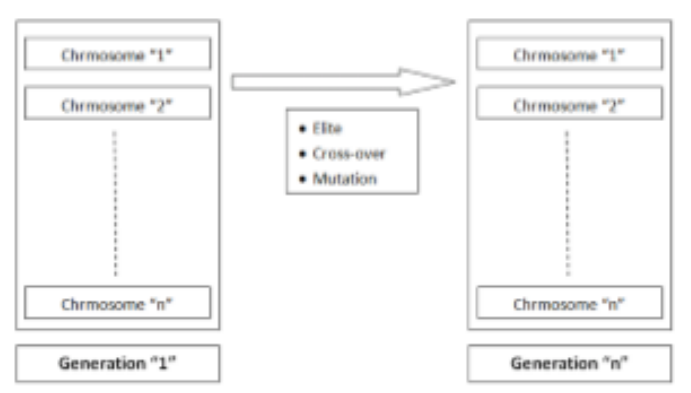

Fig. 12. Genetic algorithm process detailed

\section{1) GA chromosome identification:}

By looking at any singular configuration, we found the joints configuration will be $\mathrm{Q}=(\theta 1 \theta 2 \theta 3 \theta 4 \theta 5)$, $\theta 1$ doesn't affect the singular configuration at any point as it is responsible to rotate the robot around itself, in other words $\theta 1$ makes the robot rotate in $360^{\circ}$ around itself and at each degree there is an imaginary plane, the configuration for the elbow singularity will be repeated at each plane at each degree, same as for $\theta 5$.therefore, at any given 360 planes we can consider the arm robot as a $3 \mathrm{DOF}$ arm robot with only $(x, z, \alpha)$. Therefore the main concern is to control and optimize the three angular joints $\left(\begin{array}{lll}\theta 2 & \theta 3 & \theta 4\end{array}\right)$. Now choosing the structure and representation of each chromosome, there are two types of representation; binary and real number representation, choosing real number representation will be more efficient and a better expresses for the aspects of the fitness function. The structure of the chromosome;

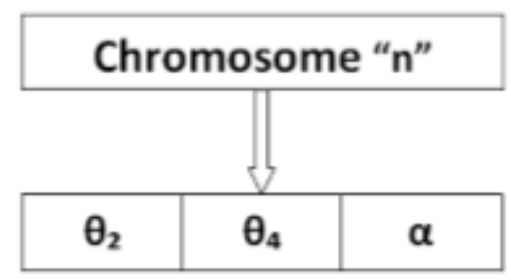

Fig. 13. Genetic algorithm process detailed

\section{2)GA fitness function:}

We will using GA to find the min distance between to joints configuration, first configuration is the one at singular point and second configuration is by shifting $\theta 3$ by $0.2 \circ$ so that is pass by the singular point without any disturbance for the system and using optimization techniques to find the minimum $\theta 2$ and $\theta 4$. the objective function that should be optimized is function defining the distance between the two cartesian locations using the two joints configuration $\mathrm{Q} 1=[\theta 2, \theta 4, \alpha]$ and $\mathrm{Q} 1=\left[\theta 2,0.2^{\circ}, \alpha\right]$. in addition to the cartesian location, the aim was to optimize the difference in the joints, so the objective function become;

$$
\begin{array}{r}
f\left(\Theta_{2}, \Theta_{4}, \alpha\right)=(\lambda) \cdot \sqrt{\left(\Delta\left(\Theta_{2}\right)^{2}+\left(\Delta\left(\Theta_{4}\right)^{2}\right.\right.}+ \\
(1-\lambda) \cdot \sqrt{\Delta(X)^{2}+\Delta(Z)^{2}+\Delta(\alpha)^{2}}
\end{array}
$$

Where;

$$
\begin{gathered}
\Delta\left(\Theta_{2}\right)=\Theta_{2}^{\prime}-\Theta_{2} \\
\Delta\left(\Theta_{4}\right)=\Theta_{4}^{\prime}-\Theta_{4} \\
\Delta(\alpha)=\alpha^{\prime}-\alpha \\
\Delta(X)=x_{2}-x_{1} \\
\Delta(Z)=\mathrm{z}_{2}-\mathrm{z}_{1}
\end{gathered}
$$

where $\mathrm{x}_{1}$ and $\mathrm{z}_{1}$ are the coordinates of the singular pose, and $\mathrm{x}_{2}$ and $\mathrm{z}_{2}$ are the coordinates of the desired optimized pose near singularity. we studied around 11000 singular points and found the optimized values which will help us to work without any errors, for three different values of $\theta_{3}$ and they were 
$0.05^{\mathrm{O}}, 0.1^{\mathrm{O}}$ and $0.2^{\mathrm{O}}$, we found that the desired optimized values for $\theta 2$ and $\theta 4$ for each case, is the same as its value in the singular configuration but minus half the value of $\theta 3$, in other words, if the value of $\theta 3$ was $0.2^{\mathrm{O}}$ then $\theta 2$ will be $\left(\theta_{2}-0.1\right)$ and $\theta 2$ will be $\left(\theta_{2}-0.1\right)$. We assumed the population size 300 individuals, number of generations 500 , and GA parameters were 0.8 for crossover, $10 \%$ elitism and 0.1 mutation. an example for results can be recognized in the following figure. The convergence of both $\theta 2$ and $\theta 4$ in addition to the

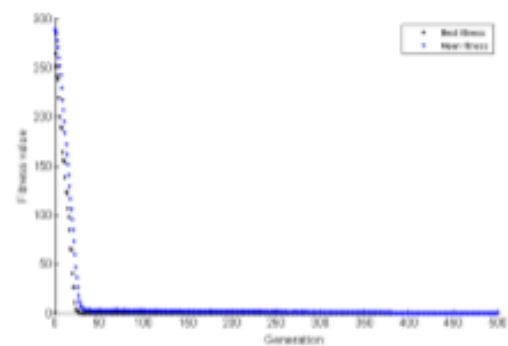

Fig. 14. the fitness function for 300 individuals and 500 generations

change in the cartesian coordinates $\mathrm{X}$ and $\mathrm{Z}$ has been tested to identify to determine the sufficient number of generations needed. the convergence of the fitness function alone was not enough to determine the total number of generations needed to find the optimum solutions, a further investigations has to be done to fine tune the results, such fine tuning was determined by investigation the change in each joint in addition to the change in the cartesian values, as shown in Fig:(15) and Fig:(16) that at generation 500 the change is becoming so trivial, if fitness function values was the only factor to be considered, iteration would have stopped at 50 generation as in Fig:(14)

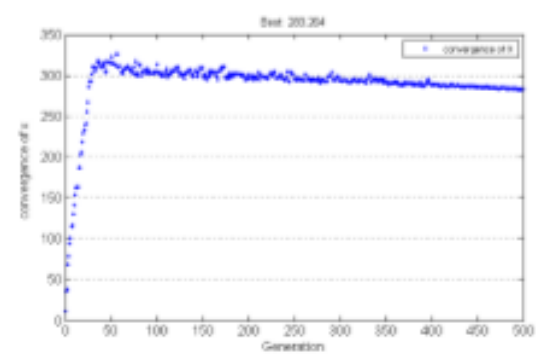

Fig. 15. The convergence of $\mathrm{X}$ coordinate with each generation

The effect of changing $\lambda$ on both cartesian error and joints error is shown in Fig:(18)

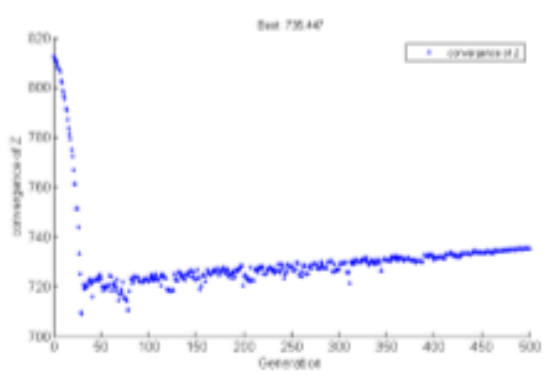

Fig. 16. The convergence of $Z$ coordinate with each generation

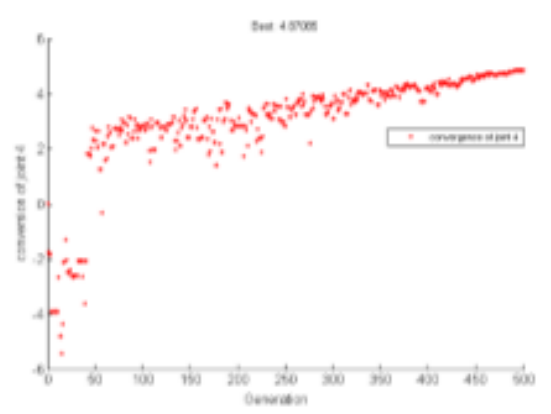

Fig. 17. The convergence of $\theta_{4}$ with each generation

it is obvious that the as $\lambda$ increased from $0-1$, the total error increased which indicates that the important parameter is to control the cartesian error through our application. also the change in both $\theta 2$ and $\theta 4$ with respect to $\lambda$ has been tested; the main effect of $\lambda$ is mainly on $\theta 4$ as $\theta 2$ doesn't change dramatically with the change in $\lambda$

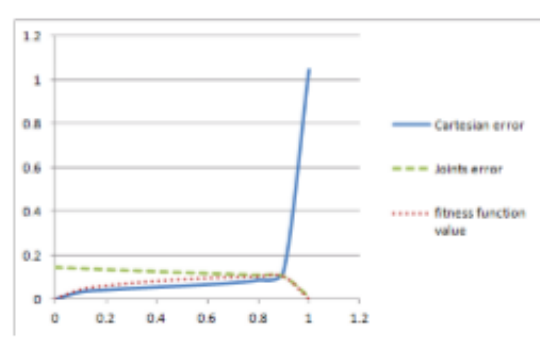

Fig. 18. The change in Error in joints,Error in cartesian coordinates and fitness function values versus change in $\lambda$

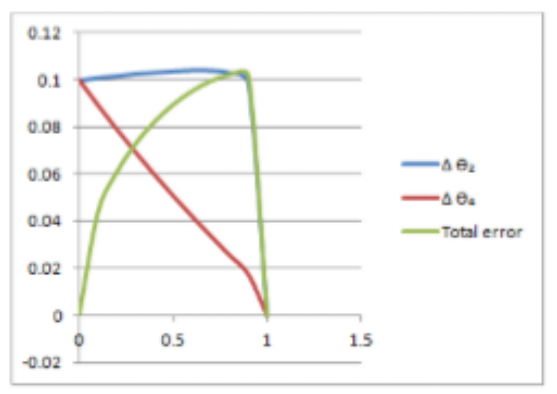

Fig. 19. The change in Error in $\theta_{2}$, change in $\theta_{4}$ and fitness function value versus $\lambda$ 


\section{Future work}

After investigating the singular points in our workspace, the next step was to try to avoid such points and find a way to work beside singular points without collision or sudden movements.

\section{References}

1. 1IFToMM Commission A, Terminology for Theory of Machines and Mechanisms, Mechanism and Machine Theory, 26, 1991

2. Z.C. Lai, and D.C.H. Yang, A New Method for the Singularity Analysis of Simple Six-link Manipulators, Int. J. of Robotics Research, 1986, 2: 66-74.

3. S.L. Wang, and K.J. Waldron, A Study of the Singular Configurations of Serial Manipulators, Pans. Of the ASME J. of Mechanisms, Pansmission, and Automation in Design, 1987, 109:14 20.

4. K.H. Hunt, A.E.Samue1, and P.R. McAree, Special Configurations of Multi-finger Multi-freedom Grippers A Kinematic Study, Znt. J. of Robotics Research, 1991 2: $123-134$.

5. J. Sefrioui, and C.M. Gosselin, Singularity Analysis and Representation of Planar Parallel Manipulators, Robotics and Autonomous Systems, 1993, 10: 209224

6. Gosselin, C. and Angeles, J., Singularity Analysis of Closed-Loop Kinematic Chains, IEEE Trans. Robotics and Automation, 6 (1990).

7. J.P. Merlet, Parallel Manipulators, Part 2: Theory, Singular Configurations and Grassmann Geometry, Technical Report No. 791, INRIA, France, 1988

8. J.P. Merlet, Singular Configurations of Parallel Manipulators and Grassmann Geometry, Int. J. of Robotics Research, 1989, 8: 45- 56.
9. Zlatanov, D., Fenton, R. G. and Benhabib, B., Singularity Analysis of Mechanisms and Robots via a MotionSpace Model of the Instantaneous Kinematics, Proc. IEEE Int. Conf. on Robotics and Automation, San Diego, CA, (1994)

10. Zlatanov, D., Fenton, R. G. and Benhabib, B., Singularity Analysis of Mechanisms and Robots via a VelocityEquation Model of the Instantaneous Kinematics, Proc. IEEE Int. Conf. on Robotics and Automation, San Diego, CA, (1994)

11. M.J.D. Hayes, M.L. Husty and P.J. Zsombormurray. Singular Configuration of Wrist-Partitioned 6R Serial Robots: a Geometric Perspective for Users,

12. D.E. Goldberg, B. Korb, and K. Deb. "Messy genetic algorithms: Motivation, analysis, and first results". Complex Systems, October 1989

13. S. Tabandeh,, C. Clark, "A Genetic Algorithm Approach to solve for Multiple Solutions of Inverse Kinematics using Adaptive Niching and Clustering", IEEE Congress on Evolutionary Computation, 2006, 16-21.

14. B. Kazem , A. Mahdi and A. Oudah, "Motion Planning for a Robot Arm by Using Genetic Algorithm", Jordan Journal of Mechanical and Industrial Engineering, 2008.

15. P. J. Fleming,R. C. Purshouse . "Genetic algorithms in control systems engineering".2001. 